\title{
Transcranial Magnetic Stimulation for the Treatment of Cocaine Addiction
}

\author{
Hanif Soomro' ${ }^{1}$, Alex O'Neill-Kerr' ${ }^{1}$, Leigh Neal ${ }^{2}$, Chris Griffiths' ${ }^{1}$, Robert De Vai ${ }^{1}$ \\ ${ }^{1}$ Northamptonshire Healthcare NHS Foundation Trust, Northampton, UK \\ ${ }^{2}$ Smart rTMS Limited, London, UK \\ Email: hanif.soomro@nhs.net
}

How to cite this paper: Soomro, H., O'Neill-Kerr, A., Neal, L., Griffiths, C., \& De Vai, R. (2020). Transcranial Magnetic Stimulation for the Treatment of Cocaine Addiction. Open Journal of Depression, 9, 26-30.

https://doi.org/10.4236/ojd.2020.92003

Received: April 23, 2020

Accepted: May 25, 2020

Published: May 28, 2020

Copyright (c) 2020 by author(s) and Scientific Research Publishing Inc. This work is licensed under the Creative Commons Attribution International License (CC BY 4.0).

http://creativecommons.org/licenses/by/4.0/

\begin{abstract}
Objective: The aim of this paper is to present the clinical data analysis results from a service delivering repetitive transcranial magnetic stimulation (rTMS) for people with cocaine-use disorder (CUD). Methods: The study was a retrospective investigation of routinely collected data on patients receiving rTMS between 2018 and 2019. Measures used were a cocaine craving Visual Analogue Scale (VAS), Hospital Anxiety and Depression Scale (HADS) and Patient Health Questionnaire (PHQ-9) self-rated depression measures. Results: The outcome data of 10 patients with CUD were analysed. There was a statistically significant reduction and a large effect size on CUD and depression scales. Conclusions: Reductions in craving and depression indicate the potential benefits to patients and to society of rTMS in treating CUD. Further sufficiently powered RCTs are warranted with studies focusing on the optimization of rTMS treatment and exploring the underlying mechanisms.
\end{abstract}

\section{Keywords}

Repetitive Transcranial Magnetic Stimulation (rTMS), Cocaine, Service Delivery, Addiction

\section{Introduction}

Cocaine is a drug that is extensively abused and is extremely addictive. Worldwide about $\mathfrak{E 6 2}$ billion of cocaine is sold each year (Centre for Research on Globalization, 2018) and around a million people in the UK take cocaine (Office for National Statistics, 2019) with up to $20 \%$ of users becoming dependent (Public Health England, 2017). There is no approved pharmacotherapy to treat addiction, and behavioural/psychological approaches have had limited success (Public Health England, 2017). 
Repetitive transcranial magnetic stimulation (rTMS) is a form of neuromodulation: a non-invasive and non-convulsive technique where a purpose-made electromagnetic coil is placed against the patient's scalp to deliver a short, powerful magnetic field pulse to induce electric currents in the cerebral cortex; this is achieved painlessly in the conscious subject (Hardy et al., 2016). rTMS is recommended for use to treat depression by United States' Food and Drug Administration (FDA) (Janica \& Dokucu, 2015) and UK's The National Institute for Health and Care Excellence (NICE, 2015). There is evidence to support the routine clinical service rTMS treatment of depression (Griffiths et al., 2019b) and anxiety (Griffiths et al., 2019a).

Evidence suggests that rTMS results in changes in brain activity, metabolism and connectivity (Kito et al., 2008). Repetitive transcranial magnetic stimulation (rTMS) stimulates parts of the brain such as the mesolimbic dopamine pathway which may be involved in craving. Findings suggest that rTMS modulates neural activity via two main mechanisms: frontostriatal glutamate-bearing afferents to medium spiny neurons of the ventral striatum, and projections from pyramidal neurons of the fifth layer of the Prefrontal Cortex ( $\mathrm{PfCx}$ ) impinging upon dopamine (DA) containing midbrain neurons, thereby inducing DA release in the nucleus accumbens. The effect of TMS to increase DA levels transiently in cortical areas and modulate reduced dopaminergic activity in the limbic system are probable mechanisms in restoring pre-drug functionality and stimulating the PfCx; functioning of the cortical network strengthened and improving executive-control circuits (Bolloni et al., 2018).

rTMS may help reduce cocaine craving and consumption (Politi et al., 2008). A systematic review (seven studies) revealed that using high-frequency TMS to treat drug addiction decreased craving level (Ma et al., 2019). Research evidence indicates the therapeutic potential of TMS as a safe and cost effective method in treating cocaine-use disorder (CUD) (Bolloni et al., 2018). rTMS treatment for addiction should be provided in multiple sessions (Ma et al., 2019). This present study retrospectively investigates routinely collected service data on CUD diagnosed patients receiving rTMS.

\section{Methods}

Age, sex at birth, craving Visual Analogue Scale (VAS), Patient Health Questionnaire (PHQ-9) and The Hospital Anxiety and Depression Scale (HADS) were collected and collated. The sample included 10 patients with a cocaine addiction, 9 of whom were male. The mean age was 35.1, with a range from 26 to 48 years. The mean number of rTMS sessions received was 12.78 . As the continuous variables were not normally distributed, Wilcoxon signed-rank tests $(Z)$ were used to compare baseline with post-treatment measures, with the calculated effect sizes. Inclusion was diagnosis with CUD and exclusion was lack capacity to consent to treatment and rTMS contraindications (history of epileptic seizures, metal implants near the head, use of imipramine, amitriptyline, 
doxepine, nortriptyline, maprotiline, chlorpromazine, clozapine, foscarnet, ganciclovir, ritonavir, amphetamines). Ethical clearance was provided by the service provider.

\section{Treatment}

CUD patients were treated with rTMS using Politi's protocol: 20 trains of 2 second $15 \mathrm{~Hz}$ with inter-train interval of 30 seconds at $100 \%$ of resting motor threshold (rMT) at the left dorsolateral prefrontal cortex (DLPFC) daily for 2 weeks. Each patient underwent rTMS using a medical device.

\section{Results}

\section{Craving Scale}

Measure employed was a craving Visual Analogue Scale (VAS) on a scale 0 10. There was a statistically significant reduction in craving scores following treatment from a mean of 7.8 to $1.6(p=0.008)$, with a large effect size $(\mathrm{r}=0.61)$.

\section{Patient Health Questionnaire}

The Patient Health Questionnaire (PHQ-9) is a self-rated depression measure (Kroenke et al., 2001). A Wilcoxon signed-rank test revealed there was a significant reduction in PHQ-9 scores following treatment from a mean of 15.8 to 2.6 $(p=0.018)$, with a large effect size $(\mathrm{r}=0.63)$.

\section{Hospital Anxiety and Depression Scale}

The Hospital Anxiety and Depression Scale (HADS) is a self-assessment measure of depression, anxiety, as well having a total score (Zigmond \& Snaith, 1983). While analysis revealed there was no statistically significant change in anxiety scores (mean of 9.4 to 4.7 ) following treatment ( $p=0.207$ ), there was a statistically significant reduction in depression scores from a mean of 8.4 to 2.0 $(p=0.027)$, with a large effect size $(\mathrm{r}=0.61)$. Total HADS scores did not significantly change (mean of 17.9 to 6.7$)$ from baseline ( $p=0.116)$.

\section{Discussion}

Encouraging results in a clinical service were observed which align with the emerging research literature of the value of rTMS for CUD. Reductions in both craving and in depression indicate the benefits to patients and therefore to society of rTMS in combating the harm caused by CUD. Clinicians and patients subjectively reported abrupt cessation of cocaine craving at around day 7 (4200 pulses).

The results show that rTMS treatment in a clinical service can be effective in treating CUD and depression in patients with CUD diagnosis, but that there is not an effect on reducing levels of anxiety. These results add to evidence that rTMS can be an effective treatment for CUD (Ma et al., 2019; Bolloni et al., 2018; Politi et al., 2008). This effect may be sustained, resulting in long-lasting reductions in cocaine use (Madeo et al., 2020). However, evidence has not as yet warranted a recommendation by FDA in the USA or NICE in the UK as a treatment for CUD. 


\section{Limitations}

Data was extracted from a clinical database and patient notes. Treatment was open label and adjunct to any existing antidepressant treatments, with the absence of a control. Data was from single site in the UK limiting generalizability, however; patients were from across the UK, partially negating this.

\section{Conclusion}

TMS could be an important clinical treatment for what is considered a difficult problem to treat. Further sufficiently powered RCTs are warranted (Bolloni et al., 2018). Future studies should focus on the optimization of this promising technique and exploring the underlying mechanisms (Ma et al., 2019).

\section{Conflicts of Interest}

The authors declare no conflicts of interest regarding the publication of this paper.

\section{References}

Bolloni, C., Badas, P., Corona, G., \& Diana, M. (2018). Transcranial Magnetic Stimulation for the Treatment of Cocaine Addiction: Evidence to Date. Substance Abuse and Rehabilitation, 9, 11. https://doi.org/10.2147/SAR.S161206

Centre for Research on Globalization (2018). Cocaine, Heroin, Cannabis, Ecstasy: How Big Is the Global Drug Trade?

Griffiths, C., O’Neill-Kerr, A., De Vai, R., \& Da Silva, K. (2019a). Impact of Repetitive Transcranial Magnetic Stimulation on Generalised Anxiety Disorder in Treatment Resistant Depression. Annals of Clinical Psychiatry, 31, e2-e7.

Griffiths, C., O’Neill-Kerr, A., Millward, T., \& Da Silva, K. (2019b). Repetitive Transcranial Magnetic Stimulation (rTMS) for Depression: Outcomes in a United Kingdom (UK) Clinical Practice. International Journal of Psychiatry in Clinical Practice, 23, 122-127. https://doi.org/10.1080/13651501.2018.1562077

Hardy, S., Bastick, L., O’Neill-Kerr, A., Sabesan, P., Lankappa, S., \& Palaniyappan, L. (2016). Transcranial Magnetic Stimulation in Clinical Practice. BJPsych Advances, 22, 373-379. https://doi.org/10.1192/apt.bp.115.015206

Janicak, P. G., \& Dokucu, M. E. (2015). Transcranial Magnetic Stimulation for the Treatment of Major Depression. Neuropsychiatric Disease and Treatment, 11, 1549. https://doi.org/10.2147/NDT.S67477

Kito, S., Fujita, K., \& Koga, Y. (2008). Changes in Regional Cerebral Blood Flow after Repetitive Transcranial Magnetic Stimulation of the Left Dorsolateral Prefrontal Cortex in Treatment-Resistant Depression. The Journal of Neuropsychiatry and Clinical Neurosciences, 20, 74-80. https://doi.org/10.1176/jnp.2008.20.1.74

Kroenke, K., Spitzer, R. L., \& Williams, J. B. (2001). The PHQ-9: Validity of a Brief Depression Severity Measure. Journal of General Internal Medicine, 16, 606-613. https://doi.org/10.1046/j.1525-1497.2001.016009606.x

Ma, T., Sun, Y., \& Ku, Y. (2019). Effects of Non-Invasive Brain Stimulation on Stimulant Craving in Users of Cocaine, Amphetamine, or Methamphetamine: A Systematic Review and Meta-Analysis. Frontiers in Neuroscience, 13, 1095.

https://doi.org/10.3389/fnins.2019.01095 
Madeo, G., Terraneo, A., Cardullo, S., Gómez Pérez, L. J., Cellini, N., Sarlo, M., Gallimberti, L. et al. (2020). Long-Term Outcome of Repetitive Transcranial Magnetic Stimulation in a Large Cohort of Patients with Cocaine-Use Disorder: An Observational Study. Frontiers in Psychiatry, 11, 158. https://doi.org/10.3389/fpsyt.2020.00158

National Institute for Health and Care Excellence (NICE) (2015). Repetitive Transcranial Magnetic Stimulation for Depression. https://www.nice.org.uk/guidance/ipg542

Office for National Statistics (2019). Drugs Misuse: Findings from the 2018/19 Crime Survey for England and Wales. London: ONS.

https://doi.org/10.1016/S1361-3723(18)30105-2

Politi, E., Fauci, E., Santoro, A., \& Smeraldi, E. (2008). Daily Sessions of Transcranial Magnetic Stimulation to the Left Prefrontal Cortex Gradually Reduce Cocaine Craving. American Journal on Addictions, 17, 345-346.

https://doi.org/10.1080/10550490802139283

Public Health England (2017). An Evidence Review of the Outcomes That Can Be EXpected of Drug Misuse Treatment in England. London: PHE.

Zigmond, A. S., \& Snaith, R. P. (1983). The Hospital Anxiety and Depression Scale. Acta Psychiatrica Scandinavica, 67, 361-370.

https://doi.org/10.1111/j.1600-0447.1983.tb09716.x 